\title{
Erratum to: Vanishing theorems on toric varieties in positive characteristic
}

\section{Qihong Xie ${ }^{1}$}

Published online: 14 March 2015

(C) Springer-Verlag Berlin Heidelberg 2015

\section{Erratum to: Math. Z. (2014) 276:191-202 DOI 10.1007/s00209-013-1193-2}

In the original publication, the arguments for Theorem 1.1 were erroneous. The corrected version is provided below.

The author proved Theorems 1.1-1.3 in [3] for not necessarily torus-invariant divisors on toric varieties in positive characteristic. Recently, an anonymous referee provided the following example to show that Theorem 1.1 does not necessarily hold for non torus-invariant divisors, which means that the arguments of [3] are erroneous.

Example 1 Let $X=\mathbb{P}^{2}, D \subseteq X$ a smooth projective curve of degree $d \geq 4$, and $L=\mathcal{O}_{X}(1)$. Then the cohomology exact sequence associated to the short exact sequence

$$
0 \rightarrow \Omega_{X}^{1} \otimes L \rightarrow \Omega_{X}^{1}(\log D) \otimes L \rightarrow \mathcal{O}_{D} \otimes L \rightarrow 0
$$

together with the standard Bott vanishing for $\mathbb{P}^{2}$ show that

$$
H^{1}\left(X, \Omega_{X}^{1}(\log D) \otimes L\right) \cong H^{1}\left(X,\left.L\right|_{D}\right) .
$$

Then the cohomology exact sequence associated to the short exact sequence

$$
0 \rightarrow \mathcal{O}_{X}(-d+1) \rightarrow \mathcal{O}_{X}(1) \rightarrow \mathcal{O}_{D}(1) \rightarrow 0
$$

shows that $H^{1}\left(X,\left.L\right|_{D}\right) \cong H^{2}\left(X, \mathcal{O}_{X}(-d+1)\right)$, which is nonzero as $d \geq 4$. Therefore $H^{1}\left(X, \Omega_{X}^{1}(\log D) \otimes L\right) \neq 0$, which implies that Theorem 1.1 in [3] does not necessarily hold for non torus-invariant divisors on toric varieties in arbitrary characteristic.

The online version of the original article can be found under doi:10.1007/s00209-013-1193-2.

\footnotetext{
Qihong Xie

qhxie@fudan.edu.cn

1 School of Mathematical Sciences, Fudan University, Shanghai 200433, China
} 
Recall that in Definition 2.3 of [3], a lifting of the relative Frobenius morphism $\widetilde{F}: \widetilde{X} \rightarrow$ $\widetilde{X}^{\prime}$ is said to be compatible with a lifting $\widetilde{D}$ of the divisor $D$ if $\widetilde{F}^{*} \mathcal{O}_{\widetilde{X}^{\prime}}\left(-\widetilde{D}^{\prime}\right)=\mathcal{O}_{\widetilde{X}}(-p \widetilde{D})$. Note that the compatibility means the equality of sheaves of ideals, but not an isomorphism of line bundles. But the author made the mistake in the proof of Theorem 3.7 (ii) of [3]: he only proved the isomorphism of line bundles, then concluded that they are compatible. In fact, Theorem 3.7 (ii) generally fails for not necessarily torus-invariant divisors on toric varieties.

Remark 2 It should be mentioned that we still have the following results in spite of the mistake above:

(1) Theorems 2.4-2.8 and Theorems 3.1-3.6 of [3] were proved for general varieties under the conditions on the liftability of divisors and the relative Frobenius morphism, and on their compatibility, therefore these results remain correct.

(2) Theorem 3.7 (ii) is valid for torus-invariant divisors on toric varieties.

As a consequence, Theorems 1.1-1.3 of [3] do hold for torus-invariant divisors on toric varieties in positive characteristic. Furthermore, by using a standard argument - the reduction modulo $p$ method (cf. [2, §6]), we can show that Theorems 1.1-1.3 of [3] also hold for torusinvariant divisors on toric varieties in characteristic zero.

Thus by means of the positive characteristic method, we can reprove the Bott vanishing theorem, the degeneration of the Hodge to de Rham spectral sequence and the Kawamata-Viehweg vanishing theorem for torus-invariant divisors on toric varieties in arbitrary characteristic, which have already been proven by Fujino [1].

Let $F$ be a perfect field (of any characteristic). We only state the revised main theorems as follows and refer to [4] for their explicit proofs.

Theorem 3 (Bott vanishing) Let $X$ be a projective toric variety over $F, D$ a reduced torusinvariant Weil divisor on $X$, and $L$ an ample invertible sheaf on $X$. Then for any $j>0$ and any $i \geq 0$,

$$
H^{j}\left(X, \widetilde{\Omega}_{X}^{i}(\log D) \otimes L\right)=0 .
$$

Theorem 4 (Degeneration of Hodge to de Rham spectral sequence) Let $X$ be a proper toric variety over $F$, and $D$ a reduced torus-invariant Weil divisor on $X$. Then the Hodge to de Rham spectral sequence degenerates at $E_{1}$ :

$$
E_{1}^{i j}=H^{j}\left(X, \widetilde{\Omega}_{X}^{i}(\log D)\right) \Longrightarrow \mathbf{H}^{i+j}\left(X, \widetilde{\Omega}_{X}^{\bullet}(\log D)\right) .
$$

Theorem 5 (Kawamata-Viehweg vanishing) Let $X$ be a projective toric variety over $F$, and $H$ a nef and big torus-invariant $\mathbb{Q}$-divisor on $X$. Then for any $i>0$,

$$
H^{i}\left(X, K_{X}+\ulcorner H\urcorner\right)=0 .
$$

\section{References}

1. Fujino, O.: Multiplication maps and vanishing theorems for toric varieties. Math. Zeit. 257, 631-641 (2007)

2. Illusie, L.: Frobenius et dégénérescence de Hodge. In: Bertin, J., Demailly, J.-P., Illusie, L., Peters, C. (eds.) Introduction à la Théorie de, Hodge Panoramas et Synthèses, vol. 3, pp. 113-168. Socité de Mathmatiques de France, Marseilles (1996)

3. Xie, Q.: Vanishing theorems on toric varieties in positive characteristic. Math. Zeit. 276, 191-202 (2014)

4. Xie, Q.: Vanishing theorems on toric varieties, with Erratum to a former paper. arXiv:1401.3067, preprint 